\title{
Prospective Validation that Vulnerable Plaque Associated with Major Adverse Outcomes Have Larger Plaque Volume, Less Dense Calcium, and More Non-Calcified Plaque by Quantitative, Three-Dimensional Measurements Using Intravascular Ultrasound with Radiofrequency Backscatter Analysis
}

\author{
Results from the ATLANTA I Study
}

Jesus G. Vazquez-Figueroa • Sarah Rinehart • Zhen Qian • Parag H. Joshi •

Abhinav Sharma • James Lee • Hunt Anderson • Laura Murrieta •

Charles Wilmer • Harold Carlson • Kenneth Taylor • William Ballard •

Dimitri Karmpaliotis - Anna Kalynych • Charles Brown III • Szilard Voros

Received: 12 March 2013 / Accepted: 8 May 2013 / Published online: 22 May 2013

(C) The Author(s) 2013. This article is published with open access at Springerlink.com

\begin{abstract}
Whether quantitative, two-dimensional, and threedimensional plaque measurements by intravascular ultrasound with radiofrequency backscatter (IVUS/VH) are different between intermediate lesions with or without major adverse cardiovascular events (MACE) is unknown. IVUS/VH-derived parameters were compared in 60 patients with an intermediate coronary lesion (40-70 \%) between lesions that did or did not result in MACE over 12 months. IVUS/VH measurements were done at the site of the minimal lumen area (MLA) and
\end{abstract}

Work was conducted while at Piedmont Heart Institute.

J. G. Vazquez-Figueroa $(\triangle)$

Atlanta Medical Center, Atlanta, GA, USA

e-mail: ggus@hotmail.com

S. Rinehart $\cdot$ Z. Qian $\cdot$ A. Sharma $\cdot J$. Lee $\cdot$ H. Anderson $\cdot$

L. Murrieta $\cdot$ C. Wilmer $\cdot$ H. Carlson $\cdot$ K. Taylor $\cdot$ W. Ballard $\cdot$

D. Karmpaliotis · A. Kalynych $\cdot$ C. Brown III

Piedmont Heart Institute, Atlanta, GA, USA

P. H. Joshi

Johns Hopkins School of Medicine, Baltimore, MD, USA

S. Voros

Stony Brook University Medical Center, Stony Brook, NY, USA

S. Voros $(\bowtie)$

Integrated Cardiovascular Research Group, Atlanta, GA, USA

e-mail: szilardvorosmd@gmail.com on a per-plaque basis, defined by $40 \%$ plaque burden. Prespecified, adjudicated MACE events occurred in 5 of 60 patients $(8.3 \%)$. MACE lesions had larger plaque burden ( $65 \%$ vs. $53 \%, p=0.004)$, less dense calcium ( $6.6 \%$ vs. $14.7 \%, p=0.05)$, and more non-calcified plaque, mostly fibrofatty kind ( $17.6 \%$ vs. $10 \%, p=0.02)$. Intermediate coronary lesions associated with MACE at 12 months have more plaque, less dense calcium, and more non-calcified plaque, particularly fibrofatty tissue by IVUS/VH.

Keywords Intravascular ultrasound · Vulnerable plaque · Cardiac events

\section{Introduction}

Atherosclerotic coronary artery disease (CAD) remains the leading cause of morbidity and mortality in the USA, and accordingly, approximately 1,350,000 patients suffer an acute coronary syndrome (ACS) each year [1]. Moreover, approximately $20 \%$ of patients who suffered an initial event will have a recurrent event within a year $[2,3]$.

Atherosclerosis progresses through a series of molecular and cellular processes resulting in a series of geometrical and compositional changes that can be detected by imaging modalities [4]. The "vulnerable plaque" paradigm hypothesizes 
that certain histopathological features of coronary atherosclerotic plaques are associated with plaque rupture [5] and include mild-moderate luminal stenosis, large plaque burden, more lipid pool and lipid-rich necrotic core, more inflammation, and less calcification. Moreover, thin-cap fibroatheromas (TCFA) are the most predominant morphology found in autopsy series of ruptured plaques [6-13]. However, prospective validation of such features in vivo has been challenging in the absence of a reliable imaging method.

Recently, gray-scale intravascular ultrasound (IVUS) and IVUS with radiofrequency backscatter analysis (IVUS/VH) have been utilized as an invasive means of visualizing human coronary arterial plaques in vivo [14]. Several retrospective IVUS/VH studies demonstrated that plaques associated with events had larger plaque burden, more necrotic core, less calcification, and the presence of IVUS/VH defined TCFA (VH-TCFA) [15]. Furthermore, a recent, large, prospective study (PROSPECT: "A prospective natural-history study of coronary atherosclerosis") utilizing three-vessel IVUS/VH based on cross-sectional luminal analysis showed that minimal lumen area (MLA) plaque burden and the presence of VH-TCFA were independent predictors of major adverse cardiovascular events (MACE) [16]. However, features of nonobstructive plaques that resulted in MACE prospectively over a 12-month period have not been investigated before in threedimension (3D) on a per-plaque basis. Accordingly, we hypothesized that plaques associated with MACE over a 12month period will have larger plaque burden, more noncalcified plaque, more necrotic core, and less dense calcium compared to lesions that were not associated with MACE, based on 3D IVUS/VH measurements on a per-plaque basis.

\section{Methods}

\section{General Study Design}

The ATLANTA (Assessment of Tissue characteristics, Lesion morphology and hemodynamics by Angiography with fractional flow reserve, intravascular ultrasound and virtual histology and Non-invasive computed Tomography in Atherosclerotic plaques) study was a prospective, single-center, investigatorinitiated study approved by the Institutional Review Board of Piedmont Healthcare. The overall study design has been published previously [15] and is shown in Fig. 1. Patients with intermediate coronary arterial lesions who presented with signs or symptoms suggestive of myocardial ischemia and who qualified for the study based on enrollment criteria were included either from the cardiac catheterization laboratory or from the cardiovascular computed tomography laboratory. Main inclusion criteria included the following: adults ages $18-70$, no known or prior $\mathrm{CAD}$, symptoms suggestive of myocardial ischemia or high likelihood of CAD, and at least one "intermediate" coronary lesion (40-70\% percent diameter stenosis based on CTA or XRA). All patients underwent invasive coronary angiography (XRA) with quantitative coronary angiography (QCA), fractional flow reserve (FFR) measurements, intravascular ultrasound (IVUS) with radiofrequency backscatter analysis (IVUS/VH), and multi-detector computed tomography (MDCT) coronary angiography (CTA) at baseline. A "study lesion" was prospectively identified in each patient, which was quantitatively evaluated by CTA, IVUS/VH, and FFR measurements. Patients were contacted 6 and 12 months after the baseline evaluation for follow-up and returned at 1 year for repeat CTA examination. The 6- and 12-month follow-up visits were conducted through telephone interviews.

\section{Study Endpoints, Event Definitions, and Adjudication Process}

The primary endpoint of the overall study was an agreement between CTA and IVUS/VH for quantitative measurements of coronary plaque and stenosis and has been published elsewhere [15]. Therefore, sample size calculation was predicated on the number of patients required to assess the agreement between CTA- and IVUS/VH-derived quantitative measurements of plaque. Although the study was not powered to predict cardiovascular events, we prospectively collected MACE in a prespecified manner. Pre-specified components of MACE included death from any cause, myocardial infarction (MI), target lesion

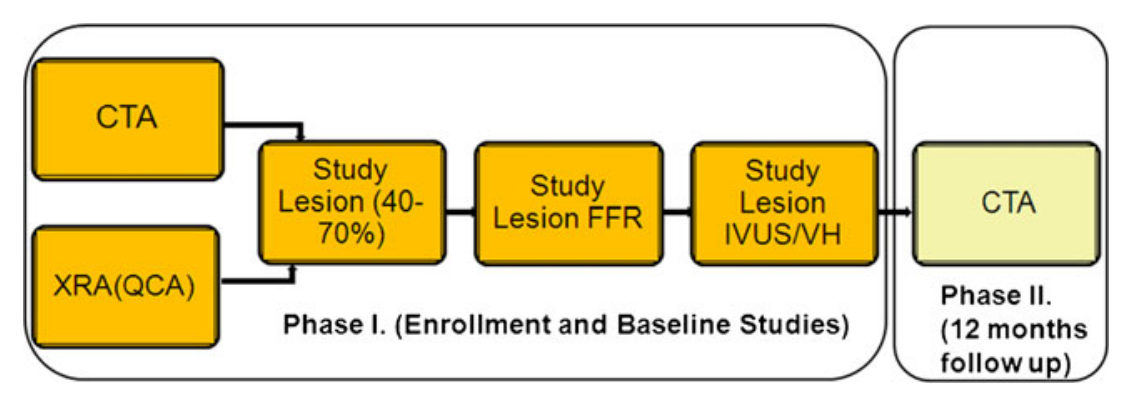

Fig. 1 ATLANTA study design. Patients with intermediate lesions (40$70 \%$ ) were recruited based on XRA or CTA; each pre-specified study lesion underwent IVUS/VH and FFR. CTA was repeated at 12 months.
$C T A$ computed tomography angiography, $X R A$ invasive $\mathrm{X}$-ray coronary angiography, $Q C A$ quantitative coronary angiography, FFR fractional flow reserve, IVUS intravascular ultrasound, $V H$ virtual histology 
revascularization (TLR), and ischemia distal to the study lesion, in a hierarchal fashion. For example, if a patient had ischemia in the territory of the study lesion that resulted in TLR, this event was only accounted for as TLR and not as TLR and ischemia. MI was defined using standard AHA/ACC definitions and included both ST-segment elevation and non-ST-segment elevation MI [17]. TLR was defined as either percutaneous coronary intervention (PCI) of the study lesion or coronary artery bypass grafting (CABG) that bypassed the study lesion. Myocardial ischemia was defined as reversible perfusion defect on radionuclide myocardial perfusion imaging, vasodilator stress cardiovascular magnetic resonance examination, or stress-induced wall motion abnormality on stress echocardiography. The AHA/ASNC 17-segment model was used to assign myocardial territories to each study lesion [18].

Each component of MACE was carefully assessed during the 6- and 12-month interviews with patients using a prospectively designed, structured questionnaire, addressing each component of the pre-specified MACE endpoint. In addition, all hospitalization records, cardiovascular procedural records, and interim office-visit records were reviewed to ensure adequate follow-up information. All cardiac catheterization images were reviewed in patients who underwent PCI or CABG during the follow-up to determine whether the study lesion was indeed revascularized. Patients who could not be contacted and who did not respond were checked against the Social Security Death Index (SSDI).

Once all information regarding cardiovascular outcomes was collected, each prospective event was carefully adjudicated by two cardiologists independently (S.V. and S.R.), and disagreement was resolved by consensus.

\section{IVUS-VH Image Acquisition}

After intracoronary injection of nitroglycerin (mean total dose per case, $561.5 \mathrm{mcg}$; range, $0-1,800 \mathrm{mcg}$ ) and after placing a guiding catheter in the target coronary artery, a 3.2-F, 20-mHz ultrasound catheter (Eagle Eye; Volcano Inc.; Rancho Cordova, CA, USA) was inserted and was advanced at least $2 \mathrm{~cm}$ beyond the most distal portion of the target lesion. Automated pullback was performed at a rate of 0.5 mm/s (R-100; Volcano Inc.; Rancho Cordova, CA, USA). The electrocardiographic signal was simultaneously recorded for the reconstruction of the radiofrequency backscatter information using In-Vision Gold (Volcano Inc.; Rancho Cordova, CA, USA).

\section{IVUS/VH Image Analysis}

De-identified IVUS/VH datasets were analyzed by an experienced cardiologist (G.V.) using dedicated software (pcVH 3.0.394, Volcano Inc., Rancho Cordova, CA, USA) on a dedicated workstation. Semi-automatic contouring of the luminal boundary and the external elastic lamina was performed in each frame. For plaque geometrical parameters, plaque burden was calculated as the difference between the vessel area and the luminal area expressed as a percentage of the vessel area (Fig. 2). Based on a previously validated algorithm [14], the software classified each pixel as dense calcium (DC; white color), fibrous tissue (FI; green color), fibrofatty tissue (FF; light green color), and necrotic core (NC; red color; Fig. 3). Total volume and percentage of each of the four components was measured in the study segment. Furthermore, we calculated the volume and percent of all non-calcified plaque components (sum of NC, FF, and FI).

Each study lesion was evaluated both in a two-dimensional (2D) and 3D fashion. Plaque classification was performed based on the plaque composition and geometrical analysis by IVUS/VH. Each plaque was characterized based on accepted IVUS/VH phenotypes such as pathological intimal thickening (PIT), thick-cap fibroatheroma (ThCFA), and TCFA $[14,19]$. PIT was defined as the presence of predominantly
Fig. 2 The figure represents the schematic for calculation of plaque burden for 2D study segment. $2 D$ bi-dimensional, EEL external elastic lamina, $I E L$ internal elastic lamina, $V$ vessel area, $L$ lumen area

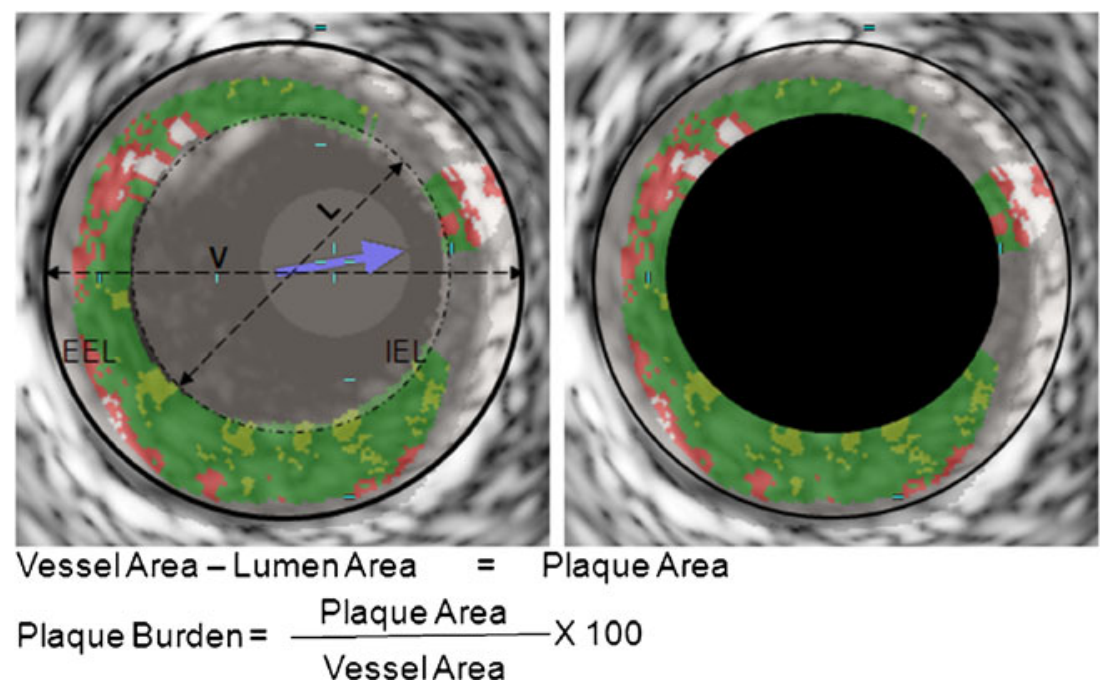



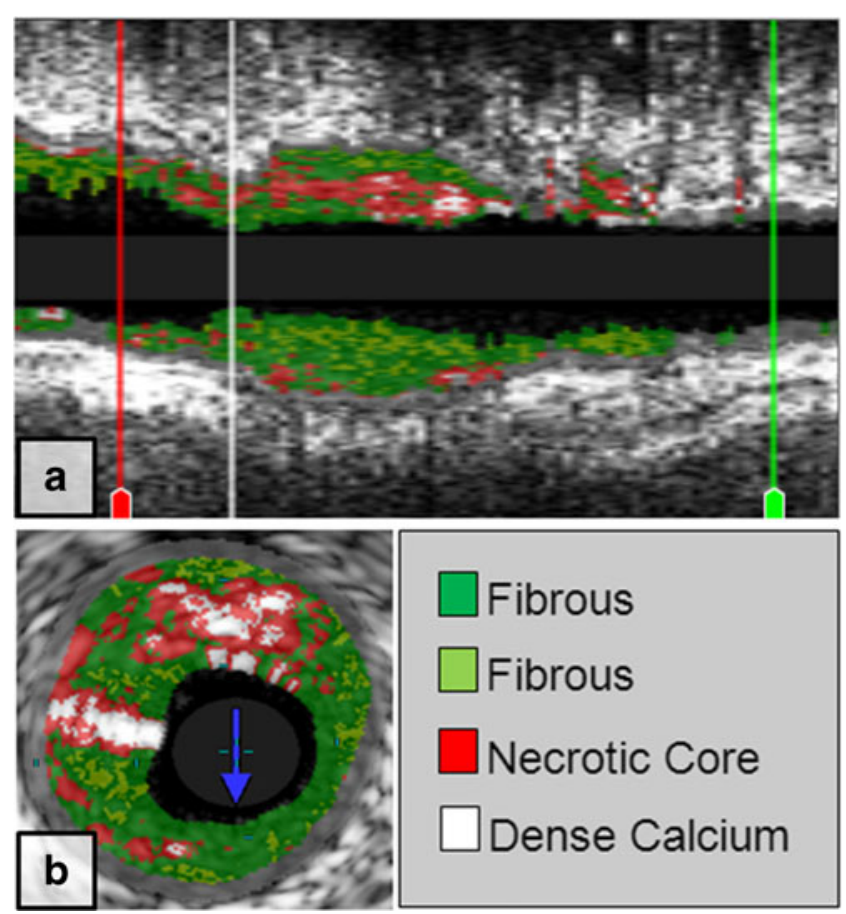

Fig. 3 Plaque composition by intravascular ultrasound with radiofrequency backscatter analysis (IVUS/VH). IVUS/VH segment is shown in the entire longitudinal section (a) and in cross-section at the minimal luminal area (MLA) frame (b)

FI and FF tissue with $\leq 10 \%$ of NC, $\leq 10 \%$ of DC, and with a plaque burden $\geq 40 \%$ in three consecutive frames. Fibroatheroma lesion was determined by a plaque burden $\geq 40 \%$ with a $\mathrm{NC} \geq 10 \%$ in three consecutive frames. The fibroatheroma lesions were classified based on the presence (VH-ThCFA) or absence (VH-TCFA) of a fibrous cap (Fig. 4a-c).

$2 D$ analysis $2 \mathrm{D}$ analysis was predicated on the site of the minimal luminal area (MLA). Plaque geometrical parameters [minimal luminal diameter (MLD), MLA, and plaque burden] were measured in the MLA frame, as well as in a frame proximal and distal to the MLA, and values from these three consecutive frames were averaged. Similarly, area and percentage of each plaque component was measured in the same three consecutive frames and were averaged between these frames (Fig. 5a).

$3 D$ analysis In addition to the $2 \mathrm{D}$ analysis, we also measured plaque geometrical and compositional parameters in each distinct plaque within the region of the entire IVUS/VH pullback. The entire vessel pullback from the IVUS/VH dataset was analyzed frame-by-frame in each subject, and the MLA frame was identified. The study segment was extended proximally and distally from the MLA frame until three consecutive frames had less than $40 \%$ plaque burden (Fig. 5b). Absolute total plaque volume was measured in each plaque, as well as the volume and percent of each of the four plaque components. Plaque burden was also calculated in 3D in the entire plaque as the difference between the vessel volume and the luminal volume expressed as a percentage of the vessel volume.

\section{Statistical Methods}

Normally distributed continuous variables are expressed as mean $\pm \mathrm{SD}$, and non-normally distributed variables as median (inter-quartile range). Normality of distribution was assessed by the Kolmogorov-Smirnoff test. Clinical features and IVUS/VH-derived plaque geometrical and compositional parameters were compared between patients with and without MACE using two-sided, unpaired Student's $t$ test for variables with normal distribution, with Kruskal-Wallis one-way analysis of variance for non-normally distributed variables and with the Fischer exact test for categorical data sets. A $p$ value of 0.05 or less was considered statistically significant. Variables that were significant in univariate analysis were entered in a multivariable stepwise logistic regression model.
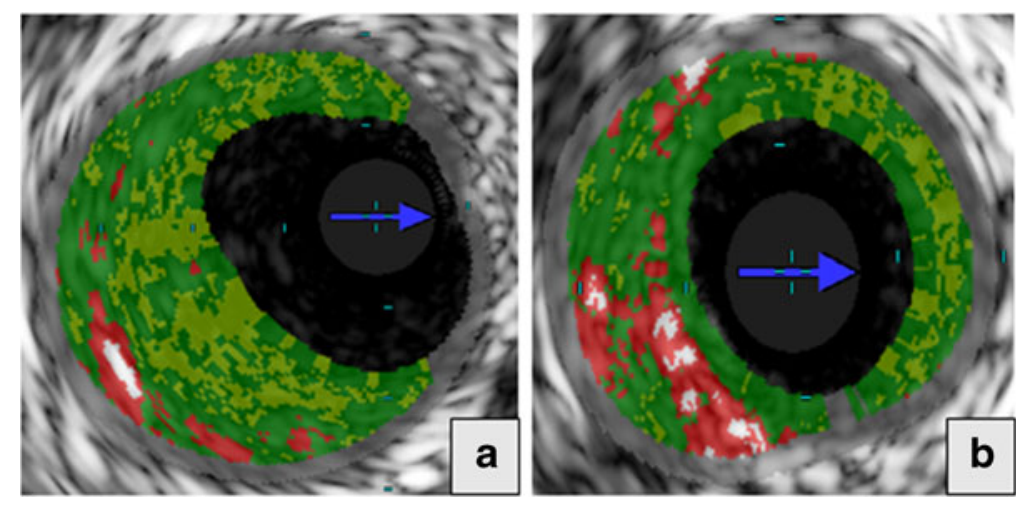

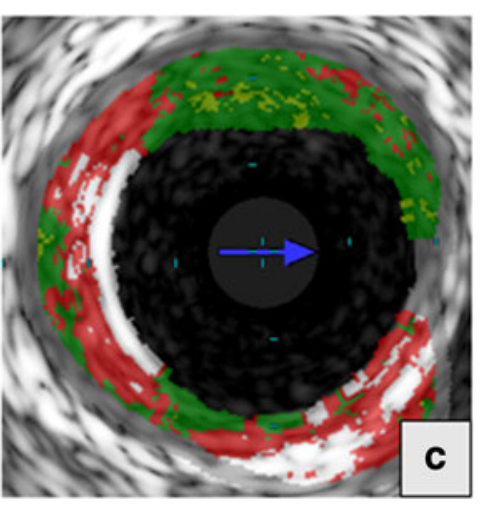

(PIT), (b) thick-cap fibroatheroma (VH-ThCFA), and (c) thin-cap fibroatheroma (VH-TCFA)
Fig. 4 Morphological lesion subtypes identified by intravascular ultrasound with radiofrequency backscatter analysis (IVUS/VH). Three plaque subtypes are shown: (a) pathological intimal thickening 
Fig. 5 Schematic

representation of $2 \mathrm{D}$ and $3 \mathrm{D}$

IVUS/VH analysis. For the 2D-

IVUS/VH analysis, plaque

geometrical parameters (MLD,

MLA, and plaque burden) were measured in the MLA frame as well as in a frame proximal and distal to the MLA, and the values from these three consecutive frames were averaged (a). For the 3D-IVUS/ $\mathrm{VH}$ analysis, in each subject, the entire vessel pullback from the IVUS/VH dataset was analyzed frame-by-frame, and the MLA frame was identified. The study segment was extended proximally and distally from the MLA frame until three consecutive frames had less than $40 \%$ plaque burden (b). $2 D$ bi-dimensional, $3 D$ three-dimensional, IVUS intravascular ultrasound, $\mathrm{VH}$ virtual histology, $M L A$ minimal lumen area
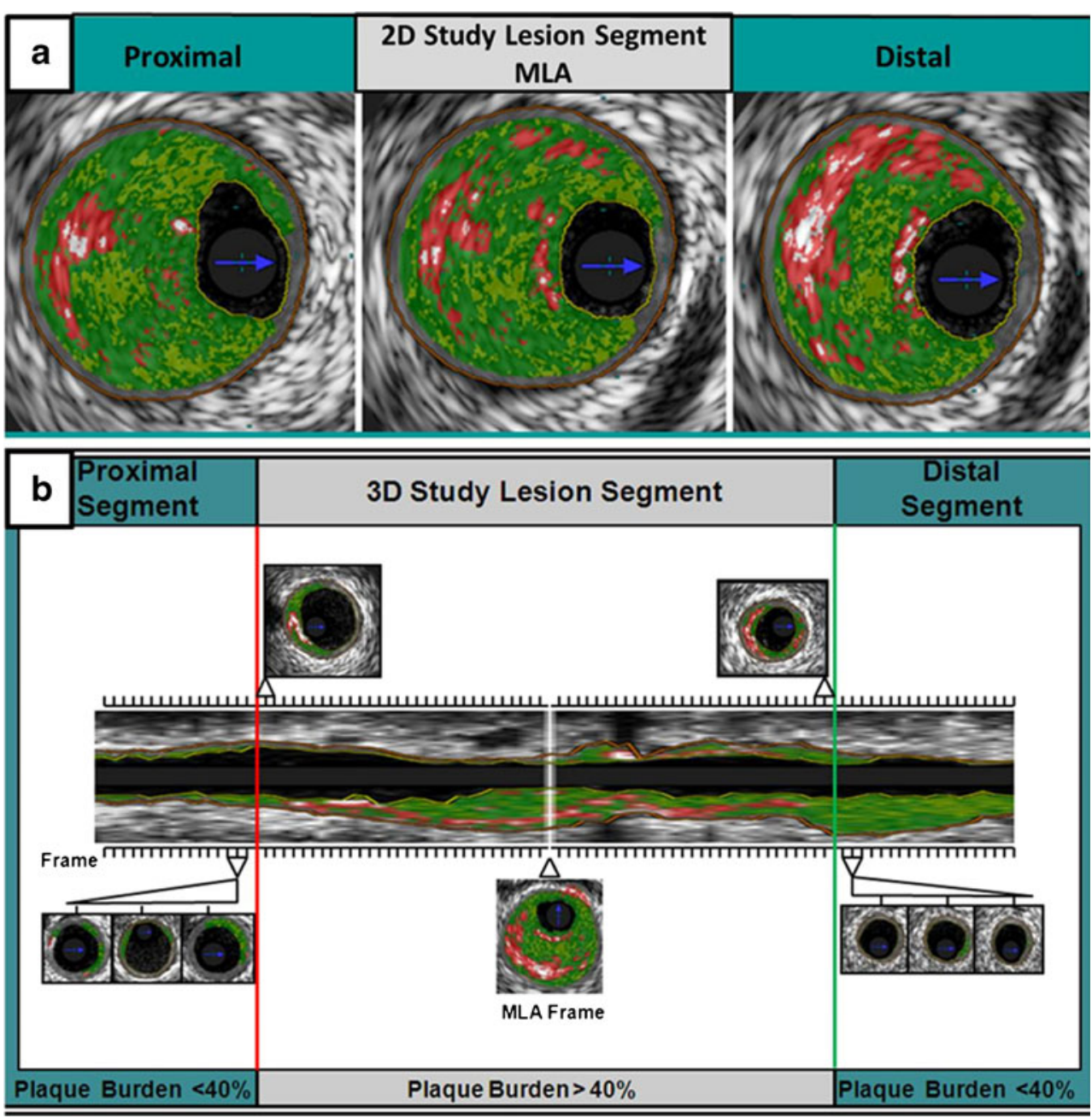

\section{Results}

General Demographics

Sixty patients were enrolled in the study; general demographic characteristics are shown in Table 1. Mean age was $60.4 \pm 7.2$ years, and $60 \%$ were men. Mean 10-year Framingham risk score in the study population was $8.4 \pm$ $6.0 \%$. The presence of hypertension, diabetes, dyslipidemia, tobacco use, and lipoprotein levels were representative for a population with no prior CAD presenting with signs and symptoms of myocardial ischemia (Table 1).

Major Adverse Cardiovascular Events (MACE)

At the end of the pre-specified 12-month follow-up, a total of five patients $(8.3 \%)$ had an adjudicated MACE. The five patients in the MACE group presented with unstable angina. There was no death or myocardial infarction. All the five MACE were in the form of target lesion revascularization; four patients $(6.7 \%)$ had PCI, and one patient $(1.7 \%)$ had
CABG. Median time to event was 72 days. IVUS/VH data was not available in one patient in the MACE group.

\section{Clinical Parameters and FFR}

The MACE and the no-MACE groups were similar with respect to age, presence of hypertension, diabetes, dyslipidemia, and tobacco use. Lipoprotein parameters were also similar between the groups (Table 2). Lesions associated with MACE were nonflow-limiting and were not different from lesions with no MACE; mean FFR in the no-MACE group was 0.89 and in the MACE group was $0.90(p=0.7)$.

IVUS/VH Parameters in the Overall Population

\section{IVUS/VH Parameters: 2D Analysis}

Geometry Geometrical parameters [MLD, MLA, percentage of area stenosis (\%AS), and percentage of diameter stenosis (\%DS)] were not different between the groups by IVUS/VH analysis (Table 3). 
Table 1 Baseline clinical characteristics

\begin{tabular}{|c|c|c|c|c|}
\hline Characteristic & $\begin{array}{l}\text { All } \\
(N=60)\end{array}$ & $\begin{array}{l}\text { MACE } \\
(N=5)\end{array}$ & $\begin{array}{l}\text { No MACE } \\
(N=55)\end{array}$ & $p$ value \\
\hline Age (years) & $60.4 \pm 7.2$ & $58.4 \pm 6.6$ & $60.6 \pm 7.3$ & 0.5 \\
\hline Men, \% & $60 \%$ & $60 \%$ & $60 \%$ & 1 \\
\hline \multicolumn{5}{|l|}{ Ethnicity } \\
\hline Caucasians & $92 \%$ & $100 \%$ & $91 \%$ & \multirow[t]{2}{*}{1} \\
\hline African-Americans & $8 \%$ & $0 \%$ & $9 \%$ & \\
\hline \multicolumn{5}{|l|}{ Risk factors } \\
\hline Hypertension & $83 \%$ & $80 \%$ & $84 \%$ & 1 \\
\hline Dyslipidemia & $95 \%$ & $100 \%$ & $95 \%$ & 1 \\
\hline Tobacco use & $58 \%$ & $60 \%$ & $58 \%$ & 1 \\
\hline Diabetes & $73 \%$ & $80 \%$ & $73 \%$ & 1 \\
\hline $\begin{array}{l}\text { Framingham risk score } \\
\text { (10-year risk })\end{array}$ & $8.5 \pm 6.0$ & $10.2 \pm 9.9$ & $8.3 \pm 5.6$ & 0.5 \\
\hline \multicolumn{5}{|l|}{ Symptoms } \\
\hline Chest pain & $45 \%$ & $60 \%$ & $44 \%$ & 1 \\
\hline Shortness of breath & $10 \%$ & $0 \%$ & $11 \%$ & 1 \\
\hline \multicolumn{5}{|l|}{ Medications } \\
\hline Aspirin & $71 \%$ & $100 \%$ & $69 \%$ & 0.3 \\
\hline Beta-blockers & $51 \%$ & $40 \%$ & $52 \%$ & 0.7 \\
\hline ACE inhibitor & $31 \%$ & $40 \%$ & $30 \%$ & 0.6 \\
\hline $\mathrm{ARB}$ & $10 \%$ & $20 \%$ & $9 \%$ & 0.4 \\
\hline Nitrate & $10 \%$ & $40 \%$ & $7 \%$ & 0.08 \\
\hline Statin & $68 \%$ & $40 \%$ & $70 \%$ & 0.3 \\
\hline $\begin{array}{l}\text { Cholesterol absorption } \\
\text { inhibitors }\end{array}$ & $25 \%$ & $0 \%$ & $28 \%$ & 0.3 \\
\hline Niacin & $15 \%$ & $20 \%$ & $15 \%$ & 0.6 \\
\hline Fibric acid derivatives & $5 \%$ & $0 \%$ & $6 \%$ & 1 \\
\hline
\end{tabular}

Normally distribute variables, mean $\pm \mathrm{SD}$

$M A C E$ major adverse cardiac events, $S D$ standard deviation

Composition 2D analysis showed significantly higher plaque area in lesions associated with MACE compared to those without MACE $\left(12.5 \pm 8.3\right.$ vs. $\left.7.9 \pm 3.5 \mathrm{~mm}^{2}, p=0.04\right)$. DC percentage was lower in the MACE group $(2.5 \pm 0.3 \%$ vs. $13.4 \pm 9.4 \%, p=0.03)$, while $\mathrm{FF}$ area and percentage were higher $\left(2.9 \pm 1.7\right.$ vs. $0.8 \pm 1.0 \mathrm{~mm}^{2}, p<0.001$, and $34.2 \pm 15.9 \%$ vs. $12.5 \pm 10.2 \%, p<0.001$, respectively). Other compositional parameters were comparable in the two groups (Table 4 and Fig. 6). Plaque type was characterized as VH-TCFA in all five patients with MACE, while only
32 of 47 patients (69\%) in the no-MACE group had this lesion type $(p=0.42)$. Other lesion subtypes in the no-MACE group were VH-ThCFA (29 \%) and PIT (2\%).

\section{IVUS/VH Parameters: 3D Analysis}

Geometry There were no differences in MLD and MLA by IVUS/VH between the MACE and no-MACE groups (Table 5).

Composition Lesions associated with MACE had significantly larger plaque burden as compared to lesions not associated with MACE $(65.1 \pm 5.1 \%$ vs. $53.0 \pm 7.8 \%, p=$ $0.004)$. FF volume and percentage were significantly higher in the MACE group as compared to the no-MACE group $\left(44.1 \pm 30.3\right.$ vs. $20.7 \pm 15.9 \mathrm{~mm}^{3}, p=0.01$, and $17.6 \pm$ $12.7 \%$ vs. $10.0 \pm 5.6 \%, p=0.02$, respectively). The MACE group had a proportionally higher amount of non-calcified plaque (NCP; $93.6 \pm 3.5 \%$ vs. $86.0 \pm 7.8 \%, p=0.05$ ). Conversely, the percentage of DC was lower in MACEassociated lesions $(6.4 \pm 3.5 \%$ vs. $14.0 \pm 7.8 \%, p=0.05)$. Results are shown in Table 6 and illustrated in Fig. 6 (bottom row).

\section{Multivariable Analysis}

Clinical features and IVUS-VH plaque characteristics were tested in a multivariable stepwise logistic regression model that included age, gender, presence of diabetes, lipoprotein parameters, and the geometrical and compositional plaque parameters as independent variables and MACE as the dependent variable. Only fibrofatty volume was retained as an independent variable with an odds ratio of 1.058 for MACE (95\% confidence interval, 1.004 to $1.115, p=0.03)$.

\section{Discussion}

There are two significant novel aspects of the present study. First, this is one of a few studies that prospectively compared coronary plaque characteristics using IVUS/VH in
Table 2 Baseline lipoprotein values

Normally distribute variables, mean \pm SD

\begin{tabular}{lllll}
\hline Characteristic & All $(N=60)$ & MACE $(N=5)$ & No MACE $(N=55)$ & $p$ value \\
\hline Laboratory values & & & & \\
TC $(\mathrm{mg} / \mathrm{dL})$ & $155.0 \pm 43.3$ & $166.0 \pm 14.9$ & $154.0 \pm 45.0$ & 0.6 \\
ApoB $(\mathrm{mg} / \mathrm{dl})$ & $78.1 \pm 27.5$ & $86.8 \pm 15.4$ & $77.3 \pm 28.4$ & 0.5 \\
LDL-C $(\mathrm{mg} / \mathrm{dl})$ & $84.7 \pm 35.7$ & $97.1 \pm 19.4$ & $83.5 \pm 36.7$ & 0.4 \\
ApoAI $(\mathrm{mg} / \mathrm{dl})$ & $144.9 \pm 30.1$ & $154.5 \pm 35.6$ & $144.0 \pm 29.8$ & 0.5 \\
HDL-C & $38.3 \pm 12.1$ & $39.2 \pm 10.9$ & $38.2 \pm 12.6$ & 0.9
\end{tabular}


Table 3 2D IVUS/VH geometrical plaque differences between MACE and no MACE groups

\begin{tabular}{llll}
\hline IVUS/VH parameter & $\begin{array}{l}\text { MACE } \\
(\text { mean } \pm \text { SD })\end{array}$ & $\begin{array}{l}\text { No MACE } \\
(\text { mean } \pm \text { SD })\end{array}$ & $p$ value \\
\hline MLD & $1.78 \pm 0.46$ & $1.96 \pm 0.48$ & 0.5 \\
MLA & $3.62 \pm 1.52$ & $4.12 \pm 2.12$ & 0.6 \\
$\% A S$ & $46.8 \pm 22.4$ & $52.7 \pm 15.8$ & 0.5 \\
$\% D S$ & $26.0 \pm 20.29$ & $31.6 \pm 11.52$ & 0.4 \\
\hline
\end{tabular}

Normally distribute variables, mean $\pm \mathrm{SD}$

IVUS intravascular ultrasound, $V H$ virtual histology, $M L D$ minimal lumen diameter, $M L A$ minimal lumen area, $\% A S$ percentage area stenosis, $\% D S$ percentage diameter stenosis, $M A C E$ major adverse cardiac events, $S D$ standard deviation

patients with MACE versus those without MACE over a 12month period. Secondly, in addition to a cross-sectional analysis at the MLA site, we also used a detailed, 3D quantitative characterization of each plaque within the region of the IVUS pullback, providing more insight into the overall features of those plaques.

The present study has five important findings. First, as expected, event rate associated with intermediate lesions was low; only 5 of 60 plaques $(8.3 \%)$ were responsible for MACE over a 12-month period. Secondly, FFR was similar in plaques with and without MACE. Thirdly, as previously reported in PROSPECT [16] and VIVA [20], lesions associated with MACE had significantly more plaque, as evidenced by higher plaque area at the MLA site and higher plaque burden in the entire plaque. Fourthly, plaque composition in MACE lesions was shifted toward less calcified and more non-calcified components, as evidenced by less dense calcium both at the MLA site as well as in the entire plaque. Finally, a unique and novel finding was the higher amount and percentage of fibrofatty tissue both at the MLA site as well as in the entire plaque. Importantly, all five MACE lesions were characterized as VH-TCFA compared to only $69 \%$ of non-MACE plaques, although this was not statistically significant.

The progression of atherosclerosis follows a reasonably predictable course [22] through some distinct phases and characteristic phenotypes and several features can be detected by IVUS/VH [21, 23]. Growth in coronary plaque size initially occurs in an outward fashion with preservation of the luminal size; this process is called "positive" remodeling and was initially described by Glagov [24] and presents an excellent target for atherosclerosis imaging by IVUS/VH [25]. This stage of positive remodeling is followed by continued phagocytosis by macrophages leading to development of a necrotic core and subsequent formation of TCFAs [6, 9]. While IVUS/VH is able to accurately detect and quantify necrotic core in human coronary arterial plaques [21], its resolution is not high enough to measure the thickness of the fibrous cap; however, an IVUS/VH definition of TCFA has been devised and is referred to as VH-TCFA [15, 26] (Fig. 5). Ruptured TCFAs are thought to be one of the most important underlying causes of sudden cardiac death and ACSs [6, 8]. Calcification, which occurs as part of the healing process in large, positively remodeled plaques that undergo repeated, spontaneous intra-plaque hemorrhage without leading to ACS [27], is also an easy target for IVUS/VH imaging [28, 29]. Finally, the latest stages of the atherosclerotic process are characterized by further calcification and fibrosis, severe luminal stenosis, and negative remodeling and can also be easily detected by IVUS/VH.

In the context of this atherosclerosis paradigm, our study showed that MACE lesions had similar lumen size and FFR compared to non-MACE lesions, while they were characterized by larger plaque burden with more non-calcified plaque, especially the fibrofatty kind, with significantly less calcium. Furthermore, all five of MACE lesions were characterized as VH-TCFA. Therefore, our study is consistent with histopathological studies of coronary atherosclerosis.

Our findings are also consistent with previous imaging studies and expand on those findings. With regards to event rates, a large meta-analysis by Nicholls et al. demonstrated that MACE rate associated with non-obstructive lesions, similar to our study, was approximately $11 \%$ per year, similar to the $8.3 \%$ found in our study [30]. The results of the large, prospective PROSPECT study have been published showing an annualized event rate of $6.9 \%$ in intermediate coronary lesions, similar to our study [16].

It is not surprising that in the present study FFR was not different between groups since we only included intermediate lesions. It is well known that with the inclusion of the entire spectrum of intermediate-to-severe stenosis, FFR has significant prognostic value [31].

Table 4 2D IVUS/VH compositional plaque differences between MACE and no MACE groups

\begin{tabular}{llll}
\hline $\begin{array}{l}\text { IVUS/VH } \\
\text { parameter }\end{array}$ & $\begin{array}{l}\text { MACE } \\
(\text { mean } \pm \text { SD) }\end{array}$ & $\begin{array}{l}\text { No MACE } \\
(\text { mean } \pm \text { SD })\end{array}$ & $p$ value \\
\hline Plaque area & $12.5 \pm 8.3$ & $7.9 \pm 3.5$ & 0.04 \\
FI area & $4.8 \pm 4.2$ & $2.7 \pm 1.7$ & 0.5 \\
FF area & $2.9 \pm 1.7$ & $0.8 \pm 0.9$ & $<0.001$ \\
NC area & $1.7 \pm 1.9$ & $1.3 \pm 1.0$ & 0.5 \\
DC area & $0.2 \pm 0.2$ & $0.7 \pm 0.6$ & 0.2 \\
FI, \% & $47.9 \pm 7.0$ & $51.1 \pm 13.9$ & 0.7 \\
FF, \% & $34.2 \pm 15.9$ & $12.5 \pm 10.2$ & $<0.001$ \\
NC, \% & $15.4 \pm 9.3$ & $23.0 \pm 10.5$ & 0.2 \\
DC, \% & $2.5 \pm 0.3$ & $13.4 \pm 9.4$ & 0.03 \\
\hline
\end{tabular}

Normally distribute variables, mean \pm SD

$2 D$ bi-dimensional, IVUS intravascular ultrasound, $V H$ virtual histology, $M A C E$ major adverse cardiac events, $S D$ standard deviation, $F I$ fibrous, $F F$ fibrofatty, $N C$ necrotic core, $D C$ dense calcium 


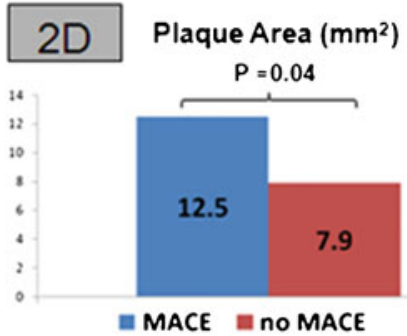

3D Plaque Burden (\%)

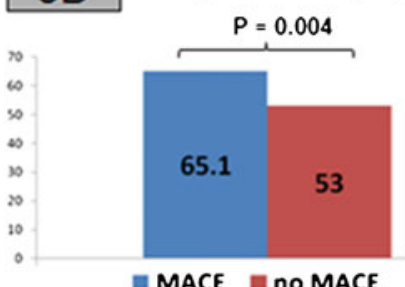

Fibrofatty area $\left(\mathrm{mm}^{2}\right)$

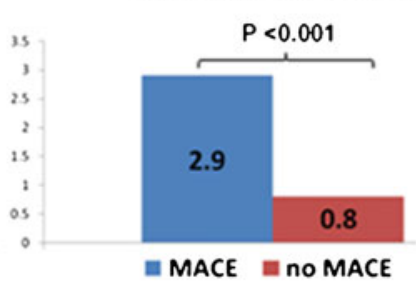

Fibrofatty Volume $\left(\mathrm{mm}^{3}\right)$

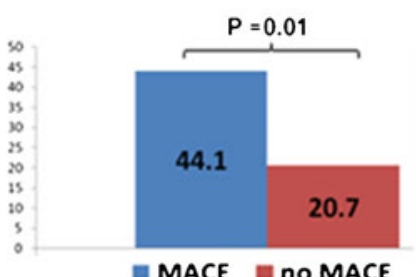

Fibrofatty $(\%)$

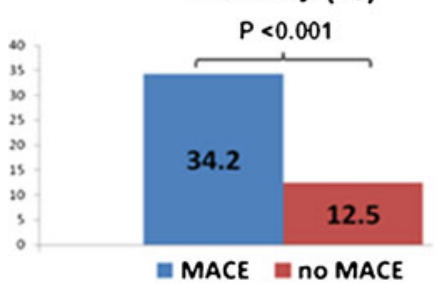

Fibrofatty (\%)

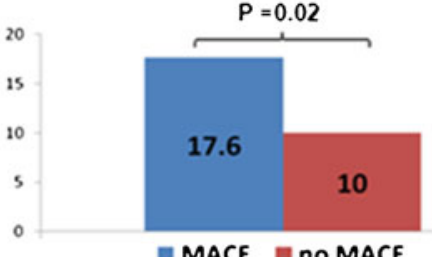

Dense Calcium (\%)

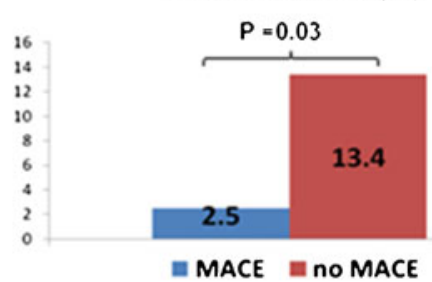

Fig. 6 Significant predictors of MACE via the two-dimensional (2D; top row) and three-dimensional (3D; bottom row) IVUS/VH analyses. MACE major adverse cardiac events, $2 D$ bi-dimensional, $3 D$ three-dimensional, $I V U S$ intravascular ultrasound, $V H$ virtual histology

Previous IVUS-based studies also showed that MACE lesions were associated with larger plaque burden and relatively preserved luminal size [30-32].

Results from PROSPECT, a large, prospective, landmark study using three-vessel IVUS/VH in approximately 700 patients with ACS followed for a median of 3.4 years, showed that $\mathrm{MLA}<4 \mathrm{~mm}^{2}$ [hazard ratio (HR), 3.21, $p=0.001$ ), plaque burden $70 \%(\mathrm{HR}, 5.03, p<0.001)$ or greater, and the presence of VH-TCFA $(3.35, p<0.001)$ were independent predictors of MACE. Results from VIVA, an additional large prospective study using three-vessel IVUS/VH in 170 patients with stable angina and ACS followed for 1.9 years, demonstrated that MLA $<4 \mathrm{~mm}^{2}$ (HR, 2.91, $p=0.036$ ), plaque burden $>70 \%$ (HR, 7.48, $p=0.001$ ), and VH-TCFA (HR, 8.16, $p=0.007$ ) were associated with MACE; our findings are largely consistent with these findings $[16,20]$.

Table 5 3D IVUS/VH geometrical plaque differences between MACE and no MACE groups

\begin{tabular}{llll}
\hline $\begin{array}{l}\text { IVUS/VH } \\
\text { parameter }\end{array}$ & $\begin{array}{l}\text { MACE } \\
(\text { mean } \pm \text { SD) }\end{array}$ & $\begin{array}{l}\text { No MACE } \\
(\text { mean } \pm \text { SD })\end{array}$ & $p$ value \\
\hline MLD & $1.78 \pm 0.46$ & $1.96 \pm 0.48$ & 0.5 \\
MLA & $3.62 \pm 1.52$ & $4.12 \pm 2.12$ & 0.6 \\
$\% A S$ & $46.8 \pm 22.4$ & $52.7 \pm 15.8$ & 0.5 \\
$\%$ DS & $26.0 \pm 20.29$ & $31.6 \pm 11.52$ & 0.4 \\
RI & $88.0 \pm 1.8$ & $88.6 \pm 9.9$ & 0.9 \\
\hline
\end{tabular}

Normally distribute variables, mean $\pm \mathrm{SD}$

$3 D$ three-dimensional, IVUS intravascular ultrasound, $V H$ virtual histology, $M L D$ minimal lumen diameter, $M L A$ minimal lumen area, $\% A S$ percentage area stenosis, $\% D S$ percentage diameter stenosis, $M A C E$ major adverse cardiac events, $R I$ remodeling index, $S D$ standard deviation
We also found that MACE lesions had more noncalcified plaque and less calcified components. This is consistent with histopathological findings, which showed that ACS lesions were associated with less calcification compared to stable lesions [11, 12]. These findings are also consistent with those by Yamagishi et al. [32] and Sano et al. [33], who also found more lipid-rich plaque and more echolucent plaque in culprit lesions, compared to non-culprit lesions.

One relatively unexpected, and previously unreported, finding in our study was the difference in fibrofatty tissue volume and percentage between MACE and non-MACE

Table 6 3D IVUS/VH compositional plaque differences between MACE and no MACE groups

\begin{tabular}{llll}
\hline $\begin{array}{l}\text { IVUS/VH } \\
\text { parameter }\end{array}$ & $\begin{array}{l}\text { MACE } \\
(\text { mean } \pm \text { SD })\end{array}$ & $\begin{array}{l}\text { No MACE } \\
(\text { mean } \pm \text { SD })\end{array}$ & $p$ value \\
\hline Plaque burden $(\%)$ & $65.1 \pm 5.1$ & $53.0 \pm 7.8$ & 0.004 \\
FI volume & $133.0 \pm 74.0$ & $106.0 \pm 70.6$ & 0.5 \\
FF volume & $44.1 \pm 30.3$ & $20.7 \pm 15.9$ & 0.01 \\
NC volume & $51.8 \pm 32.1$ & $54.2 \pm 36.0$ & 0.9 \\
DC volume & $17.3 \pm 14.2$ & $31.0 \pm 27.5$ & 0.3 \\
FI, \% & $54.6 \pm 8.1$ & $51.0 \pm 10.0$ & 0.5 \\
FF, \% & $17.6 \pm 12.7$ & $10.0 \pm 5.6$ & 0.02 \\
NC, \% & $21.3 \pm 7.1$ & $25.0 \pm 6.5$ & 0.3 \\
DC, \% & $6.4 \pm 3.5$ & $14.0 \pm 7.8$ & 0.05 \\
NCP, \% & $93.6 \pm 3.5$ & $86.0 \pm 7.8$ & 0.05 \\
\hline
\end{tabular}

All volumes are in cubic millimeters

$3 D$ three-dimensional, IVUS intravascular ultrasound, $V H$ virtual histology, $M A C E$ major adverse cardiac events, $S D$ standard deviation, $F I$ fibrous, $F F$ fibrofatty, $N C$ necrotic core, $D C$ dense calcium, $N C P$ noncalcified plaque 
lesions, both at the MLA site, as well as in the entire plaque. Interestingly, in the multivariable analysis, this was the only feature that retained independent significance. Relatively little data is available with regards to fibrofatty tissue on IVUS/VH. Histopathologically, it probably represents slightly earlier-stage, lipid-rich plaques prior to the development of a necrotic core. The fact that our study was a lower-risk population compared to some of the previous studies described above, which mostly enrolled patients with ACS, while our study population was not an ACS population may be a potential explanation for this finding. Furthermore, it has been suggested that thrombi may be identified as fibrofatty tissue on IVUS/VH [34]; however, careful review of our cases did not identify thrombus in these cases.

This study adds to the current literature on the predictive value of IVUS/VH for the prospective identification of potential lesions that may be associated with MACE in the future.

\section{Limitations}

There are several limitations to our study. Most importantly, it was a single-center study with a relatively low sample size. As it was described above, the primary endpoint of the overall study was the agreement between IVUS/VH and coronary CTA for percent atheroma volume, and sample size calculation was designed to fulfill that primary endpoint. Therefore, the study was not powered for the detection of MACE. Nevertheless, we did collect pre-specified MACE events in the study, all events were adjudicated by two cardiologists, and the statistical analysis revealed significant differences between MACE and non-MACE lesions, despite the low overall sample size. In addition, as a consequence of the lower number of patients in the MACE group, the multivariable analysis is limited. As a result, the data generated in this study should only be hypothesis generating. Secondly, the follow-up period of 12 months was relatively short, although this time-point is customary for the follow-up of MACE in large clinical trials. Thirdly, we only evaluated one vessel in each patient by IVUS/VH. Finally, we did not perform serial IVUS/VH imaging; it was only performed at baseline. Other modalities, such as coronary CTA, may be better positioned for serial imaging, as the dropout rate in serial IVUS studies is approximately 30 $40 \%$ [35].

\section{Conclusions}

In conclusion, our study found that while major cardiovascular event rates were low due to non-flow-limiting, intermediate coronary arterial lesions, MACE lesions by IVUS/VH overall had larger plaque burden due to more non-calcified plaque components, mostly due to fibrofatty tissue and less calcified components. All lesions associated with MACE within a 12month follow-up were characterized as VH-TCFA at baseline. Understanding the natural history of non-flow-limiting coronary arterial lesions will aid in designing pre-emptive strategies to combat vulnerable plaques, and such strategies will have to be tested in large-scale, multi-center clinical outcome trials.

Funding The current study (ATLANTA; NCT\# 00817102) was an investigator-initiated and investigator-sponsored study funded primarily by Abbott Vascular and Volcano, Inc., Siemens Medical Solutions, and Vital Images, and Toshiba America Medical Systems contributed partial and additional funding to the study.

Szilard Voros received research grants from Abbott Vascular, Volcano Inc., Siemens Medical Solutions, Vital Images, Toshiba America Medical Systems, Merck Inc., and Abbott Laboratories. S.V. has Speaker's Bureau, Consulting, and Advisory Board Memberships in Vital Images, Toshiba America Medical Systems, and Merck Inc. S.V. is the owner, president, and CEO of Integrated Cardiovascular Research Group, LLC; the CEO of Global Genomics Group; and the Chief Academic Officer of HDL, Inc.

Sarah Rinehart received research grants from Abbott Vascular, Volcano Inc., Siemens Medical Solutions, Vital Images, and Toshiba America Medical Systems.

Dimitri Karmpaliotis received grant support from Medtronic; D.K. has Speakers' Bureau and Consulting memberships in Abbott Vascular and Bridgepoint Medical.

Open Access This article is distributed under the terms of the Creative Commons Attribution License which permits any use, distribution, and reproduction in any medium, provided the original author(s) and the source are credited.

\section{References}

1. Writing Group, Lloyd-Jones, D., Adams, R. J., et al. (2010). Heart disease and stroke statistics-2010 update: a report from the American Heart Association. Circulation, 121, e46-e215.

2. Cannon, C. P., Braunwald, E., McCabe, C. H., et al. (2004). Intensive versus moderate lipid lowering with statins after acute coronary syndromes. The New England Journal of Medicine, 350, $1495-1504$.

3. Voros, S. (2009). "Does imaging paint a sugar-coated picture of diabetic vessels?": plaque composition in diabetics by IVUS and CT angiography. Journal of Nuclear Cardiology, 16, 339-344.

4. Muller, J. E., Tofler, G. H., \& Stone, P. H. (1989). Circadian variation and triggers of onset of acute cardiovascular disease. Circulation, 79, 733-743.

5. Virmani, R., Burke, A. P., Farb, A., \& Kolodgie, F. D. (2006). Pathology of the vulnerable plaque. Journal of the American College of Cardiology, 47, C13-C18.

6. Naghavi, M., Libby, P., Falk, E., et al. (2003). From vulnerable plaque to vulnerable patient: a call for new definitions and risk assessment strategies: part I. Circulation, 108, 1664-1672.

7. Naghavi, M., Libby, P., Falk, E., et al. (2003). From vulnerable plaque to vulnerable patient: a call for new definitions and risk assessment strategies: part II. Circulation, 108, 1772-1778.

8. Kolodgie, F. D., Burke, A. P., Farb, A., et al. (2001). The thin-cap fibroatheroma: a type of vulnerable plaque: the major precursor 
lesion to acute coronary syndromes. Current Opinion in Cardiology, $16,285-292$.

9. Stary, H. C., Chandler, A. B., Dinsmore, R. E., et al. (1995). A definition of advanced types of atherosclerotic lesions and a histological classification of atherosclerosis: a report from the Committee on Vascular Lesions of the Council on Arteriosclerosis, American Heart Association. Circulation, 92, 1355-1374.

10. Gertz, S. D., \& Roberts, W. C. (1990). Hemodynamic shear force in rupture of coronary arterial atherosclerotic plaques. The American Journal of Cardiology, 66, 1368-1372.

11. Cheng, G. C., Loree, H. M., Kamm, R. D., Fishbein, M. C., \& Lee, R. T. (1993). Distribution of circumferential stress in ruptured and stable atherosclerotic lesions. A structural analysis with histopathological correlation. Circulation, 87, 1179-1187.

12. Davies, M. J., Richardson, P. D., Woolf, N., Katz, D. R., \& Mann, J. (1993). Risk of thrombosis in human atherosclerotic plaques: role of extracellular lipid, macrophage, and smooth muscle cell content. British Heart Journal, 69, 377-381.

13. Nissen, S. E., Gurley, J. C., Grines, C. L., et al. (1991). Intravascular ultrasound assessment of lumen size and wall morphology in normal subjects and patients with coronary artery disease. Circulation, 84, 1087-1099.

14. Rodriguez-Granillo, G. A., Garcia-Garcia, H. M., Mc Fadden, E. P., et al. (2005). In vivo intravascular ultrasound-derived thin-cap fibroatheroma detection using ultrasound radiofrequency data analysis. Journal of the American College of Cardiology, 46, 2038-2042.

15. Voros, S., Rinehart, S., Qian, Z., et al. (2011). Prospective validation of standardized, 3-dimensional, quantitative coronary computed tomographic plaque measurements using radiofrequency backscatter intravascular ultrasound as reference standard in intermediate coronary arterial lesions: results from the ATLANTA I study. Journal of the American College of Cardiology: Cardiovascular Interventions, 4, 198-208.

16. Stone, G. W., Maehara, A., Lansky, A. J., et al. (2011). A prospective natural-history study of coronary atherosclerosis. The New England Journal of Medicine, 364, 226-235.

17. Thygesen, K., Alpert, J. S., \& White, H. D. (2007). On behalf of the Joint ESC/ACCF/AHA/WHF Task Force for the Redefinition of Myocardial Infarction. Universal definition of myocardial infarction. Journal of the American College of Cardiology, 50, 2173-2195.

18. Cerqueira, M. D., Weissman, N. J., Dilsizian, V., et al. (2002). Standardized myocardial segmentation and nomenclature for tomographic imaging of the heart: a statement for healthcare professionals from the Cardiac Imaging Committee of the Council on Clinical Cardiology of the American Heart Association. Circulation, 105, 539-542.

19. König, A., Margolis, M. P., Virmani, R., et al. (2008). Technology insight: in vivo coronary plaque classification by intravascular ultrasonography radiofrequency analysis. Nature Clinical Practice. Cardiovascular Medicine, 5, 219-229.

20. Calvert, P. A., Obaid, D. R., O'Sullivan, M., et al. (2011). Association between IVUS findings and adverse outcomes in patients with coronary artery disease: the VIVA (VH-IVUS in Vulnerable Atherosclerosis) Study. JACC. Cardiovascular Imaging, 4(8), 894-901.

21. Nair, A., Kuban, B. D., Tuzcu, E. M., Schoenhagen, P., Nissen, S. E., \& Vince, D. G. (2002). Coronary plaque classification with intravascular ultrasound radiofrequency data analysis. Circulation, 106, 2200-2206.

22. Tabas, I., Williams, K. J., \& Boren, J. (2007). Subendothelial lipoprotein retention as the initiating process in atherosclerosis: update and therapeutic implications. Circulation, 116, 1832-1844.

23. Nair, A., Margolis, M. P., Kuban, B. D., \& Vince, D. G. (2007). Automated coronary plaque characterisation with intravascular ultrasound backscatter: ex vivo validation. EuroIntervention, 3, $113-120$.

24. Glagov, S., Weisenberg, E., Zarins, C. K., Stankunavicius, R., \& Kolettis, G. J. (1987). Compensatory enlargement of human atherosclerotic coronary arteries. The New England Journal of Medicine, 316, 1371-1375.

25. Rodriguez-Granillo, G. A., Serruys, P. W., Garcia-Garcia, H. M., et al. (2006). Coronary artery remodelling is related to plaque composition. Heart, 92, 388-391.

26. Garcia-Garcia, H. M., Mintz, G. S., Lerman, A., et al. (2009). Tissue characterisation using intravascular radiofrequency data analysis: recommendations for acquisition, analysis, interpretation and reporting. EuroIntervention, 5, 177-189.

27. Virmani, R., Kolodgie, F. D., Burke, A. P., Farb, A., \& Schwartz, S. M. (2000). Lessons from sudden coronary death: a comprehensive morphological classification scheme for atherosclerotic lesions. Arteriosclerosis, Thrombosis, and Vascular Biology, 20, $1262-1275$

28. Wexler, L., Brundage, B., Crouse, J., et al. (1996). Coronary artery calcification: pathophysiology, epidemiology, imaging methods, and clinical implications: a statement for health professionals from the American Heart Association. Circulation, 94, 1175-1192.

29. Mintz, G. S., Popma, J. J., Pichard, A. D., et al. (1995). Patterns of calcification in coronary artery disease: a statistical analysis of intravascular ultrasound and coronary angiography in 1155 lesions. Circulation, 91, 1959-1965.

30. Nicholls, S. J., Hsu, A., Wolski, K., et al. (2010). Intravascular ultrasound-derived measures of coronary atherosclerotic plaque burden and clinical outcome. Journal of the American College of Cardiology, 55, 2399-2407.

31. Tonino, P. A. L., De Bruyne, B., Pijls, N. H. J., et al. (2009). Fractional flow reserve versus angiography for guiding percutaneous coronary intervention. The New England Journal of Medicine, 360, 213-224.

32. Yamagishi, M., Terashima, M., Awano, K., et al. (2000). Morphology of vulnerable coronary plaque: insights from follow-up of patients examined by intravascular ultrasound before an acute coronary syndrome. Journal of the American College of Cardiology, 35, 106-111.

33. Sano, K., Kawasaki, M., Ishihara, Y., et al. (2006). Assessment of vulnerable plaques causing acute coronary syndrome using integrated backscatter intravascular ultrasound. Journal of the American College of Cardiology, 47, 734-741.

34. Nasu, K., Tsuchikane, E., Katoh, O., et al. (2008). Impact of intramural thrombus in coronary arteries on the accuracy of tissue characterization by in vivo intravascular ultrasound radiofrequency data analysis. The American Journal of Cardiology, 101, 10791083.

35. Nissen, S. E. (2005). Effect of intensive lipid lowering on progression of coronary atherosclerosis: evidence for an early benefit from the reversal of atherosclerosis with aggressive lipid lowering (REVERSAL) trial [abstract]. The American Journal of Cardiology, 96, 61-68. 